\title{
Las Agencias de Calificación ¿Un Poder Financiero Fuera Del Estado? Un Análisis Desde Europa
}

\author{
As Agências de qualificação: um poder financeiro fora do Estado? \\ Uma Análise da Europa
}

Miguel J. Arjona Sánchez ${ }^{1}$

\section{RESUMEN:}

El predominio de las agencias de calificación estadounidenses ${ }^{2}$ en la economía mundial es una buena muestra del éxito de la globalización financiera, cuya influencia va más allá del marco financiero internacional, para afectar incluso al marco interno de los países occidentales. La actividad de estas agencias, regulada principalmente por la legislación norteamericana, las ha convertido en fieles guardianes de la ortodoxia financiera construida principalmente desde las instituciones, reguladores y centros académicos anglosajones ${ }^{3}$, que les ha otorgado a estos países una descomunal ventaja. Situadas en el centro del sistema financiero internacional son capaces de generar movimientos de capital proclives a los intereses que representan, actuando desde un proselitismo en favor de una globalización desarrollada en absencia de reglas multilaterales (de forma ad hoc) cuyo interés por formalizar su papel, mediante un marco jurídico internacional o cuanto menos un conjunto ordenado de reglas es cuanto menos dudoso ${ }^{4}$. Desde una inicial concepción de su actividad como meramente informativa hasta su condición de verdaderos proveedores de licencias regulatorias", lo que las convierte en instituciones (instituciones de evaluación de crédito externo" «ECAIs»), lo cierto es que estas agencias representan una aplicación extraterritorial del Derecho estadounidense en el corazón de una actividad de gran importancia estratégica para cualquier país como las finanzas, y frente al que la Unión Europea, pese a haber tomado conciencia de su situación, no ha sabido desarrollar una estrategia eficaz para defender sus intereses.

\section{ABSTRACT:}

The dominance of US rating agencies in the world economy is a good example of the success of financial globalization, whose influence goes beyond the international financial framework,

\footnotetext{
${ }^{1}$ Profesor de Derecho Constitucional, Universidad de Granada (España).

${ }^{2}$ Las principales agencias de calificación del mundo, también conocidas como las «big three» son tres firmas radicadas en la ciudad de Nueva York: Fitch Ratings, Moody's Investors Service, y Standard \& Poor's (S\&P). Por poner un ejemplo, los análisis de Moody alcanzan a ciento treinta países, 11.000 emisores corporativos, 21.000 emisores financieros públicos, y 76.000 obligaciones financieras estructuradas. Véase: MOODY'S, "Moody's Investors Services, Moody's Role in the capital markets", Moody's Web Site: (consulta: 6 de marzo de 2017): https://www.moodys.com/Pages/atc002.aspx

${ }^{3}$ Uno de los documentos más interesantes sobre el papel de este «softpower» anglosajón, incluyendo al poder académico en relación con la crisis financiera fue la película documental «Inside Job». Véase: AUDRY MARRS y CHARLES FERGUSON (Productores), CHARLES FERGUSON (Director), 2010, Inside Job, Estados Unidos: Sonny Classics. En relación con el papel del académico, véase el siguiente clip. Inside Job clip - Mishkin Looks Bad. Disponible en Internet (consulta: 8 de enero de 2017): http://www.imdb.com/videoplayer/vi3193018905?ref_=tt_pv_vi_aiv_1

${ }^{4}$ RAWI ABDELAL, Capital Rules, The construction of Global Finance, Harvard University Press, Cambridge (Massachusetts-US) and London (UK), 2007, p. 9.
} 
to affect even the internal framework of Western countries. The activity of these agencies, regulated mainly by US law, has made them faithful guardians of financial orthodoxy built primarily from Anglo-Saxon institutions, regulators and academic centres, which has given these countries a huge advantage. Located at the centre of the international financial system, they are capable of generating capital movements that are prone to the interests they represent, acting from a proselytism in favour of a globalization developed in the absence of multilateral rules (ad hoc) whose interest in formalizing their role, through an international legal framework or at least an orderly set of rules is the least doubtful. From an initial conception of their activity as merely informative to their status as true providers of regulatory licenses, "which makes them institutions (external credit evaluation institutions" "ECAIs"), the truth is that these agencies represent an extraterritorial application of US law at the heart of an activity of great strategic importance for any country such as finance, and against which the European Union, despite having become aware of its situation, has failed to develop an effective strategy to defend its interests.

\section{LA PIRÁMIDE MONETARIA Y LOS REQUERIMIENTOS INTERNACIONALES DE CAPITAL}

\subsection{El fin del patrón oro y la clasificación de las agencias}

Aunque para la ortodoxia económica el dinero siempre ha estado unido a ciertos metales preciosos que lo respaldan y a los que representa, incluso desde esta perspectiva está plenamente admitido que el devenir histórico lo ha ido alejando de lo que se denomina su reserva metálica. Este hecho se ha representado a través de una simbólica pirámide monetaria, en cuya base se sitúa el dinero con mayor respaldo metálico y por lo tanto más seguro, y sobre ella, en sucesivas capas, el de mayor riesgo. Según la ortodoxia económica, el dinero base (denominado M0 o dinero de Alta Frecuencia) está compuesto por las reservas que respaldan su reproducción, como el oro cuando se opera bajo su patrón. Sobre esta base se sitúan otras formas dinerarias de menor confianza, como el dinero fraccionario y el bancario, que tratan de ir completando de forma elástica, las enormes necesidades monetarias características de la economía capitalista. Esta concepción refleja de forma bastante gruesa el paradigma clásico sobre el dinero basado en su concepción metalista, que sin embargo no es unánime, ya que otras 
escuelas defienden su concepción endógena, basada en la autoridad del Estado (como el chartalismo o la Teoría Económica Moderna) ${ }^{5}$.

En cualquier caso, la demanda de capital propia de las sociedades capitalistas es saciada a través de una multiplicación monetaria que está basada en la deuda ${ }^{6}$, lo que abrirá la puerta a una labor de calificación de las distintas formas del dinero, que enjuiciarán la calidad de los títulos, préstamos, descuentos y anticipos de los propios bancos comerciales ante el banco central. Inicialmente percibidos como meras informaciones financieras, pasado el tiempo serán percibidos como datos relevantes sobre los que descansará el apalancamiento monetario. Esta labor, que en Europa será habitualmente llevada por los bancos centrales, será encargada en los EE.UU. a las agencias de calificación que adquirirán así un estatuto que podríamos definir como público $^{7}$, al considerarse poco apropiado que quien deba guiar la política monetaria (el banco emisor) enjuicie el valor de los empréstitos.

Este protagonismo no fue lineal, sino que se aceleró con la modificación de las constituciones económicas de los países occidentales. Un importante primer hito sería el de la generalización de las operaciones realizadas en el mercado abierto, que pasaría a partir de entonces a ser central en la financiación de los gobiernos; una decisión, que progresivamente iría sustituyendo los sistemas tradicionales de señoreaje, y acarreará dos importantes consecuencias. La primera de ellas, de orden económico, la de una mayor financiarización de la economía; y la segunda, de carácter más jurídico, la de relativizar la condición como libre de riesgo de los pagarés gubernamentales, abriendo en consecuencia, un nuevo, lucrativo e influyente mercado para las agencias (la información y calificación financiera de las deudas soberanas) lo que en un contexto de progresivo endeudamiento de los Estados en el mercado internacional de capitales transformará el mundo de las finanzas. El segundo hito sería aún más

\footnotetext{
${ }^{5}$ Ajena al metalismo para atribuir su creación a través de los tributos

${ }^{6}$ "Without disturbance to this definition, we can draw the line between "money" and "debts" at whatever point is most convenient for handling a particular problem. For example, we can treat as money any command over general purchasing power which the owner has not parted with for a period in excess of three months, and as debt what cannot be recovered for a longer period than this; or we can substitute for "three months" one month or three days or three hours or any other period; or we can exclude from money whatever is not legal tender on the spot. It is often convenient in practice to include in money time-deposits with banks and, occasionally, even such instruments as (e.g.) treasury bills. As a rule, I shall, as in my Treatise on Money, assume that money is coextensive with bank deposits». JOHN MAYNARD KEYNES, The General Theory of Employment, Interest and Money, En su «Chapter 13, The General Theory of the rate of interest», University of Missouri-Kansas City. Disponible en Web (consulta 18 de enero de 2017):http://www.umkc.edu/umkcsearch/?cx=008281657408603500330\%3Avpif2cmpa14\&cof=FORID\%3A10\&ie=UTF-8\&q=keynes

${ }^{7}$ Es decir, serán corporaciones privadas que tienen atribuidas funciones públicas.
} 
profundo, al menos en cuanto a la concepción monetaria, y se referirá a la desaparición formal del patrón oro que transcurriría durante el «Nixon Shock». A partir de ese momento, toda la producción monetaria se convertirá en un mero numerario basado en la deuda, cuya fiabilidad sería evaluada por las agencias de calificación. En ambos casos las consecuencias para la soberanía monetaria serían devastadoras, ya que las decisiones económicas de los Estados pasarían a estar bajo supervisión del sector privado de un país extranjero, principalmente del hegemón financiero del mundo.

\subsection{Basilea y los requerimientos internacionales de capital}

Tras la gran crisis del 29 aumentó notablemente la preocupación sobre el respaldo financiero de las instituciones bancarias, de la que surgieron los requisitos mínimos de capital para ser miembros del sistema de la Reserva Federal, impuestos a través de la «Reserve Act of $1913 »^{8}$. Pese a las consecuencias de esta crisis, este tipo de requerimientos no se trasladó a una normativa internacional hasta mucho tiempo después, cuando la preocupación porque la competencia condujera a una erosión del capital de los principales bancos internacionales derivó en el Acuerdo Basilea I o de "Convergencia internacional de medición de capital y estándares de capital" ${ }^{9}$. Un acuerdo - como los que vendrían después - que estaría basado en la determinación del capital que sería exigido a las entidades bancarias y que sería "establecido por el regulador con el objeto de minimizar el riesgo de quiebra y los problemas de riesgo sistémico" ${ }^{10}$. Dado el conocimiento de las agencias sobre la situación de los bancos, este nuevo requerimiento les permitirá convertirse en una importante referencia de información, aumentando su influencia.

Tras la crisis del 2005, en Basilea II se determinaría un nuevo marco regulatorio, que discriminaría las calificaciones internas, de las calificaciones asignadas por lo que se

\footnotetext{
${ }^{8}$ DE MIGUEL-DOMÍNGUEZ, JOSÉ CARLOS, al., "La medición del riesgo de crédito y el nuevo acuerdo de capital del Comité de Basilea", Publicación en Universidad de Santiago de Compostela, 2003, p. 1-21, p. 3.

${ }^{9}$ Ibíd. El texto del Acuerdo puede visitarse en la Web del Banco de Basilea: Disponible en Web (consulta 10 de febrero de 2017): http://www.bis.org/publ/bcbs04a.htm

${ }^{10}$ DE MIGUEL-DOMÍNGUEZ, JOSÉ CARLOS, al., "La medición del riesgo de crédito y el nuevo acuerdo de capital del Comité de Basilea", Op. Cit., p. 5.
} 
denominarían "instituciones de evaluación de crédito externo" («ECAIs») ${ }^{11}$, a través de las que los bancos podrían calcular sus activos. Los supervisores podrían reconocer a las Agencias de Calificación Crediticia si su metodología de calificación cumpliera con distintos requisitos de objetividad. Así se establecería en la Unión Europea a través de la Directiva sobre los requisitos de capital, que sería desarrollada y aclarada a través de las Directrices sobre el reconocimiento de las instituciones de evaluación externa de crédito («ECAI») emitidas el 20 de enero de 2006 por el Comité de Supervisores Bancarios (CEBS), que incluiría el reconocimiento mutuo. Esta nueva regulación significaría un avance respecto a la regulación de la actividad de las agencias, aunque las principales instituciones que alcanzarían este estatus volverían a ser las grandes agencias calificadoras mostrando su importancia ${ }^{12}$.

\section{LA NATURELEZA JURÍDICA DEL PODER DE LAS AGENCIAS}

\subsection{Dos fuentes de autoridad}

La influencia de las agencias proviene de dos actividades de naturaleza muy distinta. La primera surge del contenido informativo de sus calificaciones, y está basada en su "autoritas" como árbitro independiente y objetivo, que evalúa las probabilidades de impago de las obligaciones de los organismos, según los riesgos asociados a sus políticas económicas, a la luz

\footnotetext{
${ }^{11}$ PATRICK VAN ROY, "Credit ratings and the standardised approach to credit risk in Basel II", European Central Bank, Working Paper Series $\mathrm{n}^{\circ}$ 517, August 2005, p. 5. Disponible en Web (consulta 10 de febrero de 2017): http://www.ecb.europa.eu/pub/pdf/scpwps/ecbwp517.pdf

12 «ECAI recognition is a prerequisite for banks being able to use the agency's ratings to calculate their riskweighted assets in accordance with the Capital Requirements Directive. A CRA may be recognized by supervisors if its rating methodology meets the following requirements: ratings must be objective-the methodology used must, in particular, be systematic and subject to some form of validation; the process should be free from political influence and economic pressure; ratings should be reviewed at least once a year; and general information about the methodology should be documented and publicly available» SIEGFRIED UTZIG, "The financial crisis and the regulation of credit rating agencies: A European banking perspective", ADBI working paper series, Asian Development Bank Institute, Tokio, No. 188, 2010, p. 9. Disponible en Web (consulta 10 de diciembre de 2016): http://hdl.handle.net/10419/53684 http://www.adbi.org/workingpaper/2010/01/26/3446.credit.rating.agencies.european.banking/ En la Web del Banco Central Europea pueden consultarse las agencias que han obtenido la condición de «ECAI» en Europa. Disponible en Web (consulta 10 de febrero de 2017): https://www.ecb.europa.eu/paym/coll/risk/ecaf/html/index.en.html Sobre las «ECAI», véase la Web de la Autoridad Bancaria Europea. Disponible en Web (consulta 10 de febrero de 2017): https://www.eba.europa.eu/regulation-and-policy/external-credit-assessment-institutions-ecai
} 
de la ortodoxia económica-financiera que ellas mismas contribuyen a fijar ${ }^{13}$. Desde ese punto de vista, las agencias actúan como medios de comunicación, cuyas opiniones (expresadas a través de las calificaciones) deberían estar de acuerdo con la reputación del capital analizado, de modo que su negocio consistiría en la mera venta de información ${ }^{14}$.

Sin embargo, su segunda fuente de poder es mucho más controvertida, y proviene de su incorporación a la regulación financiera de los Estados Unidos, y por ardid de la globalización a las de muchos otros países alrededor del mundo ${ }^{15}$. Una vez que se incorporaron las clasificaciones a la reglamentación financiera, las agencias pasaron de vender únicamente información "a expender valiosos derechos de propiedad asociados al cumplimiento de esa regulación" ${ }^{16}$. Desde entonces, los balances de las empresas, las entidades financieras (tanto públicas como privadas), e incluso las haciendas y tesoros públicos de regiones, estados, etc. comenzaron a depender de sus clasificaciones. Lo cierto es que cuando las autoridades estadounidenses delegaron sus responsabilidades regulatorias a las agencias, mediante el uso de sus calificaciones como puntos de referencia para la exposición del riesgo crediticio al público, dotaron a sus opiniones de un carácter vinculante (con fuerza de ley) en los Estados Unidos, y mediante su incorporación a los reglamentos financieros internos en muchos otros países ${ }^{17}$. Fruto de esa expansión, un ridículo número de agencias se convirtieron legalmente en los "guardianes" de la vasta inversión estadounidense y su influencia ${ }^{18}$, expresando su interpretación sobre la marcha de la política económica de otros gobiernos o incluso bloques comerciales que - como la Unión Europea - se han habituado a convivir con sus dictámenes,

\footnotetext{
${ }^{13}$ CHRISTOPHER M BRUNER y ABDELAL RAWI, "To judge Leviathan: sovereign credit ratings, national law, and the world economy", Journal of Public Policy 25.02, 2005; p. 191-217, p. 192. Disponible en Web (consulta 13 de diciembre de 2016): https://www.cambridge.org/core/journals/journal-of-public-policy/article/divclasstitleto-judge-leviathan-sovereign-credit-ratings-national-law-and-the-worldeconomydiv/25F0DE0339EF16349B5E2E517D5DA6A9

${ }^{14}$ FRANK PARTNOY, "The Siskel and Ebert of financial markets: two thumbs down for the credit rating agencies." Washington University Law Quarterly Vol. 77, 1999, p: 619-712, p. 682. Disponible en Web (consulta 10 de diciembre de 2016): https://papers.ssrn.com/sol3/papers.cfm?abstract_id=167412

${ }^{15}$ CHRISTOPHER M BRUNER y ABDELAL RAWI, "To judge Leviathan: sovereign credit ratings, national law, and the world economy.", Op. Cit., p. 192.

${ }^{16}$ FRANK PARTNOY, "The Siskel and Ebert of financial markets: two thumbs down for the credit rating agencies." Op. Cit., p. 682.

${ }^{17}$ RAWI ABDELAL, Capital Rules, The construction of Global Finance, Op. Cit., p. 8.

${ }^{18}$ CHRISTOPHER M BRUNER y ABDELAL RAWI, "To judge Leviathan: sovereign credit ratings, national law, and the world economy.", Op. Cit., p. 192.
} 
pese a la percepción - manifestada en sede parlamentaria - de su enorme falta de comprensión de la realidad ${ }^{19}$.

\subsection{Agencias privadas con funciones públicas}

Uno de los aspectos más controvertidos de las agencias es su carácter privado, dado que su actividad afecta no sólo al sector privado, sino a la propia soberanía de los Estados. En realidad, ambas esferas están estrechamente relacionadas entre sí, en virtud del denominado "techo soberano", por el que los emisores de deuda privada no pueden obtener calificaciones crediticias, superiores a las de su país de origen (salvo raras excepciones) ${ }^{20}$. Ello redunda en una doble amenaza para el poder público, que no sólo recibe la presión de los mercados, sino las de su propio sector privado, directamente interesado en presionar al poder público, para que siga la política económica difundida desde las propias agencias, puesto que de ello depende la calificación de sus bonos. Por último, no podemos pasar por alto que, aunque estas prácticas prueban la posición adquirida por el sector privado desde el inicio de la globalización, no puede dejar de afirmarse que su poder procede de las autoridades, que lo han promovido o consentido de forma más o menos explícita ${ }^{21}$. La academia ha argumentado que las agencias de calificación evitan la intrusión del gobierno en un campo como el del análisis de valores, en el que los reguladores federales no estarían tan bien capacitados para su análisis y su actividad sería más $\operatorname{costosa}^{22}$. Sin embargo, otras razones también parecen aflorar para comprender su utilidad. Así su apariencia de neutralidad facilita su penetración exterior, lo que genera una simbiosis que retroalimenta la hegemonía financiera estadounidense, y la de las propias agencias, y cuyo "efecto colateral" es la exportación de su fuerza legal fuera del territorio estadounidense ${ }^{23}$. Paradójicamente, quizás el momento en el que mejor se ha podido desvelar dicho juego, tuvo lugar con la decisión de Standard \& Poor's, el cinco de agosto de 2005 de rebajar la clasificación

\footnotetext{
19 Ibid.

${ }^{20}$ Ibid.

${ }^{21}$ R. ABDELAL y BRUNER, "Private Capital and Public Policy: Standard \& Poor's Sovereign", Credit Ratings. Harvard Business School Case No. 9-705-027, 2005.

${ }^{22}$ FRANK PARTNOY, "The Siskel and Ebert of financial markets: two thumbs down for the credit rating agencies.", Op. Cit., p. 681.

${ }^{23}$ Obviamente lo decimos de un modo sarcástico ya que dicha extraterritorialidad de la normativa financiera (cada vez peor disimulada) resulta a fin de cuentas fundamental para el funcionamiento del engranaje construido entre el sector financiero estadounidense y las agencias.
} 
del bono del Tesoro Público estadounidense, por primera vez en la historia. En realidad, la decisión no mermaba lo más mínimo el valor refugio del bono federal, y no porque la calidad de este no se hubiera deteriorado según la ortodoxia, sino por su condición central en el sistema financiero internacional ${ }^{24}$; revelando una verdad a voces, aquella de que la credibilidad de la agencia también depende de la credibilidad de quien le atribuye su "licencia regulatoria". Como afirmó el premio Nobel en Economía, Paul Krugman: "Escuetamente, S \& P realmente está haciendo cosas - después de la debacle hipotecaria -, a las que verdaderamente no tiene derecho. Así que es indignante - no porque América sea A-OK - sino porque esa gente no está en condiciones de juzgarlo"25.

\section{LA APLICACIÓN EXTRATERRITORIAL DEL DERECHO}

\subsection{El origen de las Agencias}

Por lo tanto, y pese al valor de sus informaciones ${ }^{26}$, estos aspectos ayudan a explicar el incremento de la importancia de la calificación crediticia durante la década de 1930 hasta la actualidad $^{27}$. Sin embargo, para comprender mejor su papel es necesario volver la mirada al origen de estas instituciones financieras, porque nos dará perspectiva sobre que empresas han sido, que tipo de ordenamiento ha regulado su actividad, y en qué situación nos encontramos.

La primera calificación de bonos por parte de una agencia tuvo lugar en 1909, cuando el fundador de una de las grandes agencias (John Moody) comenzó calificando los bonos de los ferrocarriles estadounidenses, extendiendo luego su actividad a los de los servicios públicos e

\footnotetext{
${ }^{24}$ Lo que en términos económicos se denomina eufemísticamente liquidez.

${ }^{25}$ «In short, S\&P is just making stuff up — and after the mortgage debacle, they really don't have that right. So this is an outrage - not because America is A-OK, but because these people are in no position to pass judgment». PAUL KRUGMAN, "S\&P and the USA", The Conscience of a Liberal, New York Times, 5 de agosto de 2011. Disponible en Web (consulta 14 de diciembre de 2016): http://krugman.blogs.nytimes.com/2011/08/05/sp-andthe-usa/?smid=tw-NytimesKrugman\&seid=auto

${ }^{26}$ Aunque francamente muy mermado a raíz de un largo historial de malas previsiones en casos como el de ENRON.

${ }^{27}$ FRANK PARTNOY, "The Siskel and Ebert of financial markets: two thumbs down for the credit rating agencies.", Op. Cit., p. 681-682.
} 
industriales ${ }^{28}$. No fue casualidad que esta innovación procediera del mundo de los ferrocarriles. Si bien es cierto que el mercado internacional de bonos durante el siglo XIX era principalmente de deudas soberanas (la mayoría de las empresas europeas satisfacían sus necesidades de capital mediante préstamos bancarios y emisiones de acciones) ${ }^{29}$, la envergadura de la inversión para la construcción de los ferrocarriles estadounidenses requirió de tales recursos, que desarrolló por primera vez en el mundo, un enorme mercado - tanto doméstico como internacional - de bonos, que multiplicó con creces cualquier otro ${ }^{30}$. Su nacimiento se fecha en la década de 1850 , pero la aparición de las agencias de calificación se haría esperar hasta $1909^{31}$, ya que durante ese tiempo la información sobre los bonos sería suministrada desde otras instancias. En realidad, las primeras agencias eran en cierto modo una fusión de actividades hasta entonces desempeñadas separadamente ${ }^{32}$. En origen, la información financiera era una actividad muy artesanal, que a menudo procedían de las cartas de recomendación de algún reputado comerciante, banquero o abogado, con el que se hubieran relacionado previamente ${ }^{33}$. Esa fue la ascendencia de las «Credit-Reporting Agencies» especializadas en los informes de crédito, una actividad bien conocida, y que comenzó en 1841, con la fundación de la primera agencia mercantil que se dotó de una red de agentes que suministraban información a sus suscriptores ${ }^{34}$. Lo cierto es que estas agencias evolucionaron con el tiempo hacia su fusión con el negocio de la calificación de bonos, pero si bien se unieron bajo el mismo techo corporativo, funcionaron - aparentemente - como organizaciones independientes ${ }^{35}$. La segunda actividad de información financiera que terminaría cristalizando en las agencias sería la clásica actividad periodística, que de nuevo tuvo como protagonista al sector ferroviario. Los ferrocarriles, quizás por la gran extensión de sus actividades (que operaban con profesionales distribuidos a lo largo del territorio) generaron una publicación propia («The American Railroad Journal») que a partir de

\footnotetext{
${ }^{28}$ RICHARD CANTOR, FRANK PACKER, "The Credit Rating Industry", FRBNY Quarterly Review, SummerFall 1994, p. 1-26., p. 2.

${ }^{29}$ RICHARD SYLLA, "A Historical Primer on the Business of Credit Ratings." Op. Cit., p. 5.

${ }^{30}$ Ibid., p. 6.

${ }^{31}$ Ibid., p. 7.

${ }^{32}$ HERWIG M. LANGOHR, PATRICIA T. LANGOHR, The Rating Agencies and their Credit Ratings What They Are, How They Work and Why They Are Relevant, Vol. 510. John Wiley \& Sons, 201, p. 376.

${ }^{33}$ RICHARD SYLLA, "An historical primer on the business of credit rating.", Ratings, rating agencies and the global financial system. Springer US, 2002, p. 19-40, p. 7.

${ }^{34}$ Ibid., p. 7 y 8.

${ }^{35}$ Banco de Pagos Internacionales, Comité de Supervisión Bancaria de Basilea Working Papers (No. 3, agosto de 2000), Ratings de Crédito y Fuentes Complementarias de Información de Calidad Crediticia."] En: RICHARD SYLLA, "A Historical Primer on the Business of Credit Ratings." Op. Cit., p. 8. HERWIG M. LANGOHR, PATRICIA T. LANGOHR, The Rating Agencies and their Credit Ratings What They Are, How They Work and Why They Are Relevant, John Wiley \& Sons Ltd, Chichester, 2008, p. 375.
} 
1849 se especializaría en recopilar y publicar información sistemática sobre la propiedad ferroviaria, sus activos, pasivos y ganancias; y que tras la guerra civil (en 1868) comenzó a divulgar estadísticas financieras y de funcionamiento del sector durante varias décadas ${ }^{36}$. La última función que confluyó en las agencias fue la de los banqueros de inversión, que jugándose su reputación en cada transacción - suscribían, compraban y distribuían valores de las compañías ferroviarias. Esta actividad planteaba ciertos problemas de incompatibilidad, por la delicada naturaleza del negocio bancario, que podía hacer uso de información privilegiada, lo que dio lugar en la década de 1930, a las leyes de divulgación obligatoria para los emisores de valores, y la fundación de la «Securities and Exchange Commission» (muy semejante a nuestra Comisión Nacional del Mercado de Valores) ${ }^{37}$.

\subsection{Las licencias regulatorias}

Para las agencias estadounidenses, los años treinta fueron una década prodigiosa, en la que por primera vez la Reserva Federal hizo uso de las clasificaciones para examinar los bonos. En concreto fue el «Federal Reserve Bank of New York» quien ideó un sistema denominado de "ponderación de deseabilidad", que expresaba conjuntamente dos características - en principio tan diferentes - como la "seguridad" y la "conveniencia", que usaba en el análisis de los activos de los bancos miembros ${ }^{38}$. A su vez, los Estados designaron a ciertos valores como inversiones denominadas "legales", que debían formar parte de la cartera de las cajas de ahorro y los fondos fiduciarios, pese a que otras (considerados incluso valores de alta cualificación) permanecían vedadas ${ }^{39}$. En la construcción del sistema de la Reserva Federal, el poder para dirigir la tenencia de bonos jugó desde el principio un papel crucial, y con ello

\footnotetext{
${ }^{36}$ RICHARD SYLLA, "A Historical Primer on the Business of Credit Ratings." Op. Cit., p. 8 y 9. HERWIG M. LANGOHR, PATRICIA T. LANGOHR, The Rating Agencies and their Credit Ratings What They Are, How They Work and Why They Are Relevant, Op. Cit., p. 375.

${ }^{37}$ RICHARD SYLLA, "A Historical Primer on the Business of Credit Ratings." Op. Cit. 9 y 10. En la página Web de la «Securities and Exchange Commission» puede accederse a los textos de estas leyes, en concreto, la «Securities Act of 1933», la «Securities Exchange Act of 1934», la «Trust Identure Act of 1939», la «Investment Company Act of 1940», y la «Investment Advisers Act of 1940»."The Laws That Govern the Securities Industry", US Securities and Exchange Commission Web Site. Disponible en Web (consulta 4 de febrero de 2017): https://www.sec.gov/about/laws.shtml\#secact1933

38 GILBERT HAROLD, Bond ratings as an investment guide: An apraisal of their effectiveness, Columbia University, 1987. En: FRANK PARTNOY, "The Siskel and Ebert of financial markets: two thumbs down for the credit rating agencies." Op. Cit. p. 687.

${ }^{39}$ GILBERT HAROLD, Bond ratings as an investment guide: An appraisal of their effectiveness. Ronald Press Company, 1938, p. 300 y 18-19. En: Ibid., p. 687.
} 
aumentó la importancia de las clasificaciones. Dicho rol derivaba de la propia capacidad de la Junta de la Reserva Federal, para imponer las condiciones para la participación en el sistema, entre las que estaba considerar la situación financiera, y el carácter general de la administración de cada entidad, que "debe en todo momento dirigir sus negocios y ejercer sus poderes teniendo debidamente en cuenta la seguridad de sus depositantes" ${ }^{40}$. Pero el principal hito, que incorporó a las calificaciones a la normativa estadounidense tuvo lugar en 1931, y provino del Controlador de la Moneda, una institución del Departamento del Tesoro dedicada a la supervisión de las entidades financieras "analizando las carteras de los préstamos y las inversiones, la gestión de fondos, el capital, las ganancias, la liquidez, la sensibilidad al riesgo de mercado y otros aspectos relativos a identificar el riesgo de las entidades"41: "El Controlador determinó que los bonos clasificados como BBB (o equivalentes) y los superiores podrían ser contabilizados al costo, pero los bonos con clasificaciones más bajas requerían bajas fraccionarias" ${ }^{42}$. A esta norma pronto le acompañarían otras, como aquella según la cual los bancos nacionales sólo podrían comprar valores con la clasificación de "valores de inversión" (párrafo 7 de la Sección 5136 de los Estatutos Revisados de los Estados Unidos, enmendado por la Sección 308 de la Ley Bancaria de 1935), luego ampliada a los bancos estatales (Sección 9 de la Ley de la Reserva Federal $)^{43}$. El 15 de febrero de 1936 el Controlador resolvió que "en virtud de la autoridad conferida al Contralor de la Moneda se prohibía la compra de "valores de inversión” en los que las características de inversión sean clara y predominantemente especulativas, o bien sean "valores de inversión" designados con un estándar inferior a aquellos que sean clara y predominantemente especulativos" 44 . El efecto más inmediato de la resolución fue de carácter cuantitativo, la automática reducción del número de bonos negociados públicamente ${ }^{45}$, pero sobre todo tuvo un efecto cualitativo y de gran relevancia jurídica, el de convertir a las agencias,

\footnotetext{
${ }^{40}$ FRANK PARTNOY, "The Siskel and Ebert of financial markets: two thumbs down for the credit rating agencies." Op. Cit. p. 687.

${ }^{41}$ Office of the Comptroller of the Currency- US Department of the Treasury. Disponible en Web (consulta 21 de enero de 2017): https://www.occ.treas.gov/ https://www.occ.treas.gov/about/what-we-do/mission/indexabout.html

${ }^{42}$ GILBERT HAROLD, Bond ratings as an investment guide: An apraisal of their effectiveness, Op. Cit., p. 2627. En: FRANK PARTNOY, "The Siskel and Ebert of financial markets: two thumbs down for the credit rating agencies." Op. Cit. p. 687. RICHARD CANTOR, FRANK PACKER, "The Credit Rating Industry", Quarterly Review, Summer-Fall, 1994, p. 1-26, p. 6.

${ }^{43}$ GILBERT HAROLD, Bond ratings as an investment guide: An apraisal of their effectiveness, Op. Cit., p. 2728. En: FRANK PARTNOY, "The Siskel and Ebert of financial markets: two thumbs down for the credit rating agencies." Op. Cit. p. 687.

${ }^{44}$ FRANK PARTNOY, "The Siskel and Ebert of financial markets: two thumbs down for the credit rating agencies", Op. Cit., p. 688.

${ }^{45}$ Ibid., p. 688.
} 
en fuentes de autoridad respecto de los bonos, generalizando su actividad, que pasó a ser previa a las emisiones ${ }^{46}$. Con estas medidas, que hay que entender enmarcadas dentro de la prudencia bancaria que propugnaba el «New Deal», las agencias cambiaron considerablemente su naturaleza jurídica, ya que su actividad dejó de ser principalmente la de proveer información financiera fiable (sujeta por lo tanto al derecho a la información), para convertirse en proveedores de unas licencias regulatorias ${ }^{47}$ muy valiosas para la Reserva Federal.

\subsection{Organizaciones con Reconocimiento Nacional para Clasificaciones Estadísticas}

La segunda edad dorada de las clasificaciones comenzó al calor de las crisis crediticias de los setenta, a raíz de que en 1973, mediante la Regla 15c3-1, la «U.S. Securities and Exchange Commission» ${ }^{48}$ incorporara las calificaciones crediticias de las agencias a la normativa monetaria estadounidense, aprobando la consideración de algunas de dichas agencias como «Nationally Recognized Statistical Ratings Organizations» o «NRSROs» (Organizaciones con Reconocimiento Nacional para Clasificaciones Estadísticas) ${ }^{49}$; para ello se hizo uso de normas y reglamentos de calificación crediticia promulgados en virtud de la Ley de Valores de 1933, la Ley de Intercambio de Valores de 1934 y la Ley de Sociedades de Inversión de $1940^{50}$. El reconocimiento formal de dicha clasificación no fue precisamente inocuo, sino que tuvo importantes consecuencias: En primer lugar, la Comisión reconocía "de forma ilimitada, la utilidad de la clasificación estadística reconocida nacionalmente como base para establecer una línea divisoria entre los valores con mayor o menor grado de volatilidad en el mercado"51; es decir, les atribuía la capacidad de generar licencias calificatorias. En 1975, la «Securities and Exchange Commission» aplicó por primera vez la denominación «NRSRO»a las agencias, al referirse a las calificaciones crediticias que pudieran ser usadas en la

\footnotetext{
${ }^{46}$ Ibid., p. 689.

${ }^{47}$ Ibid., 690.

48 «The mission of the U.S. Securities and Exchange Commission is to protect investors, maintain fair, orderly, and efficient markets, and facilitate capital formation». U.S. Securities and Exchange Commission. Disponible en Web (consulta 22 de enero de 2017): https://www.sec.gov/about/whatwedo.shtml

${ }^{49}$ FRANK PARTNOY, "The Siskel and Ebert of financial markets: two thumbs down for the credit rating agencies.", Op. Cit., p. 690.

${ }^{50}$ Ibid., p. 691.

${ }^{51}$ «The Commission to a limited extent has also recognized the usefulness of the nationally recognized statistical rating organizations as a basis for establishing a dividing line for securities with a greater or lesser degree of market volatility). See Notice of Revision Proposed Amendments to Rule 15c3-1 under the Securities Exchange Act of 1934, Release No. 34-10,525, 1973 SEC LEXIS 2309 (Nov. 29, 1973)». En: Ibid.
} 
determinación de los requerimientos netos de capital por los corredores financieros, una denominación que posteriormente sería usado por otros reguladores distintos a la SEC, e incluso por las sociedades de inversión privada ${ }^{52}$. De hecho, en la actualidad, la mayoría de las clasificaciones (dependientes de los Estados Unidos) sólo son admitidas si son emitidas por una «RRSO» ${ }^{53}$.

Cuando se utilizó por primera vez la denominación «NRSRO», la «Securities and Exchange Commission» era consciente de que se refería tan sólo a aquellas agencias con presencia nacional, a las que se les atribuía en exclusividad licencia calificatoria. Ese puñado de agencias (S \& P, Moody's, Duff \& Phelps y Fitch) había conseguido una distinción que les convertía ipso facto en guardianes de la volatilidad de los bonos, generando un oligopolio de incalculable poder, que se mantiene hasta nuestros días ${ }^{54}$. Muy pocas serían las agencias que lograrían posteriormente dicha cualificación ${ }^{55}$, prácticamente todas estadounidenses ${ }^{56}$, aunque llame poderosamente la inclusión final de una firma japonesa, y de dos países con los que EEUU comparte su Área de Libre Comercio, como Canadá y México ${ }^{57}$. Dada la tradición de reglas antimonopolio en el Derecho estadounidense, y el privilegio de la consideración, parece difícil considerar una mera casualidad, tan reducido número de agencias reconocidas como «NRSROs», y bien podría ser el resultado de una decisión consciente de la «SEC», para favorecer una relación simbiótica entre los principales actores financieros estadounidenses, entre los que sin duda se encuentran las agencias. A favor de esta interpretación, está el hecho de que los procedimientos y condiciones exigidos por la «SEC», para conceder a las agencias su condición de «NRSRO» no sean muy explícitos: Una vez presentada la solicitud, los

\footnotetext{
${ }^{52}$ RICHARD CANTOR, FRANK PACKER, "The Credit Rating Industry", Op. Cit., p. 8.

${ }^{53}$ Ibid.

${ }^{54}$ FRANK PARTNOY, "The Siskel and Ebert of financial markets: two thumbs down for the credit rating agencies", Op. Cit., p. 691.

${ }^{55}$ Las agencias consideradas como «Nationally Recognized Statistical Ratings Organizations (NRSROs)» pueden consultarse en la página Web de la «Securities and Exchange Commission». Disponible en Internet (consulta 25 de enero de 2017): https://www.sec.gov/ocr/ocr-current-nrsros.html\#ambest

${ }^{56}$ «In 1982, Duff and Phelps received designation, followed by IBCA and Thomson Bank Watch in 1991 and 1992, respectively. The designation of the latter two has been limited to their ratings for banks and financial institutions only. In 1983, the SEC granted NRSRO status to McCarthy, Crisanti, and Maffei, however, this company's credit rating franchise was acquired by Duff and Phelps in 1991. At least six foreign rating agencies currently have applications outstanding with the SEC for designation as NRSROs». RICHARD CANTOR, FRANK PACKER, "The Credit Rating Industry", Quarterly Review, Summer-Fall, 1994, p. 1-26, p. 8.

${ }^{57} \mathrm{La}$ agencia es 1 Japan Credit Rating Agency, Ltd. Sobre la historia de esta compañía véase su propia página Web. Disponible en Web (consulta 25 de enero de 2017): https://www.jcr.co.jp/en/service/company/ En cuanto a la firma canadiense esta es DBRS, Inc, y la firma mexicana es HR Ratings de México, S.A. de C.V. Sobre la DBRS, Inc véase (consulta en Internet el 25 de enero de 2017): http://www.dbrs.com/about, y sobre HR Ratings de México SA de CV, véase (consulta en Internet el 25 de enero de 2017): http://www.dbrs.com/about)
} 
funcionarios de la «Securities and Exchange Commission» llevarán a cabo una investigación sobre el «background» del solicitante ("historia, propiedad, empleados, recursos financieros, políticas y procedimientos internos" $)^{58}$, pero sobre todo estudiarán que la agencia esté "reconocida nacionalmente por los usuarios predominantes de las calificaciones en los Estados Unidos, como emisora de calificaciones creíbles y confiables" (SEC 1994a) ${ }^{59}$; un requisito que por sí favorece a los operadores tradicionales ${ }^{60}$. La comprobación es informal, y se lleva a cabo mediante encuestas, procedimiento que se legitima con el argumento de que quien otorga dicha condición debe ser el mercado y no las autoridades, que deben limitarse a su mera comprobación $^{61}$. Dada la implicación de tantos intereses es difícil mantener que la opinión expresada sea realmente libre, y no esté sujeta a todo tipo de presiones, subjetividades, e incluso autocensura $^{62}$.

Fueron muchos las ventajas que reportaban estas clasificaciones. Por ejemplo, la «Rule 2a-7» de la Ley de Sociedades de Inversión de 1940 acreditaba a los "Organismos Estadísticos Nacionales Reconocidos", para realizar clasificaciones que determinaran las inversiones permisibles a los fondos del mercado monetario, o le otorgaran una serie de "privilegios"63. Tan sólo dos ejemplos: la «Rule 3a-7» excluye ciertas financiaciones estructuradas de la Ley de Sociedades de Inversión, si al menos una «NRSRO» no las califica en una de las dos categorías más altas ${ }^{64}$; y la «Rule 10f-3» establece una exención, que permite a las empresas de inversión comprar bonos municipales suscritos por una filial durante el período de suscripción, si hubieran sido calificados como "aptos para la inversión" («investment grade») o con uno de los tres «ratings» más altos, al menos por una «NRSRO», siempre que el municipio contara con tres años de antigüedad ${ }^{65}$. Este tipo de uso de las "Organizaciones con Reconocimiento Nacional para Clasificaciones Estadísticas" y sus "privilegios" han ido incrementándose; por ejemplo la «Rule 10b-6» prohíbe a cualquier persona que participe en una distribución de valores comprar o hacer una oferta por cualquier valor de esa clase hasta que se complete su

\footnotetext{
${ }^{58}$ RICHARD CANTOR, FRANK PACKER, "The Credit Rating Industry", Op. Cit., p. 8.

${ }^{59}$ SEC 1994a: U.S Securities and Exchange Commission. 1994 a.

${ }^{60}$ RICHARD CANTOR, FRANK PACKER, "The Credit Rating Industry", Op. Cit., p. 8.

61 Ibid.

${ }^{62}$ RAÚL ROJAS SORIANO, Investigación social: Teoría y práxis, Plaza y Valdés, México, 2007. p. 97-98.

63 FRANK PARTNOY, "The Siskel and Ebert of financial markets: two thumbs down for the credit rating agencies", Op. Cit., p. 691.

${ }^{64}$ RICHARD CANTOR, FRANK PACKER, "The Credit Rating Industry", Op. Cit., p. 7.

65 FRANK PARTNOY, "The Siskel and Ebert of financial markets: two thumbs down for the credit rating agencies." Op. Cit. p. 691.
} 
participación en la distribución; sin embargo, en 1983, la «SEC» adoptó una excepción a la regla para los títulos de deuda no convertibles que - entre otras cosas - recibieran la calificación de grado de inversión por al menos un «NRSRO» ${ }^{66}$. Por otra parte, el estatus de «NRSRO» sería utilizado en numerosas regulaciones bancarias, como los Reglamentos de la Reserva Federal, que las señalan como evaluadoras de la condición financiera de los bancos, así como una multitud de otras normas financieras, relativas a los seguros, las garantías hipotecarias, etc ${ }^{67}$. Por contra, y pese a su inflación normativa, sólo serían citadas jurisprudencialmente en tres casos federales, y en todos ellos de forma tangencial (en 1989 durante el caso SEC v. Drexel Burnham Lambert Inc., en 1995 en Heiko v. FDIC, y en 1996 durante UBS Asset Mgmt. v. Wood Gundy Corp. $)^{68}$. Hay que añadir, que la condición de "Organizaciones con Reconocimiento Nacional para Clasificaciones Estadísticas" conllevó algunos reconocimientos no sólo relativos al valor de la actividad de las agencias, sino a su propia responsabilidad de la que quedo exenta en su condición de experto establecida en la Sección 11 de la «Securities Act» (véase el punto 10 c de la regla $\mathrm{S}-\mathrm{K})^{69}$; una cuestión no exenta de polémica que no debería predicarse de todas sus actividades. Por último y de forma sorprendente, la «SEC» no exige que las «NRSRO» tengan estándares de calificación uniformes, y se refieren directamente a sus niveles de calificación, sin tener en cuenta las diferencias entre las agencias. Esto es consecuencia de la inexistencia de procedimientos específicos que regulen su $\operatorname{actividad}^{70}$,

\footnotetext{
66 Ibid.

${ }^{67}$ Ibid.

68 Ibid.

69 «349. The term NRSRO is defined and used in numerous banking regulations. See, e.g., 12 U.S.C. § 1831e (1996) (activities of savings associations); 12 U.S.C. $\$ 4519$ (1996) (authority to provide for review of enterprises by rating organization); 15 U.S.C. $\S 78 \mathrm{c}(41)$ (1996) (definition of the term "mortgage related security"). The Federal Reserve Board regulations (Reg. T) rely on NRSRO status. See 12 C.F.R. § 704.2, 704 App. A (1992). The Federal Reserve Board also relies on the term "bank rating agencies" in Regulation F, which permits banks to rely on such agencies to assess the financial condition of or to select a correspondent if the bank's board of directors has reviewed the assessment and selection criteria used by the rating agencies. See 12 C.F.R. $\S \S 206.3,206.5$ (1992). The Secondary Mortgage Market Enhancement Act of 1984 (SMMEA) required that a mortgage-related security be rated in one of the top two categories by at least one NRSRO. See 15 U.S.C. § 78c(a)(41) (1994). The Federal Deposit Insurance Act defines permissible activities of a savings association to include "investment grade" instruments, meaning an instrument rated in one of the top four categories by at least one NRSRO. See 12 U.S.C. $\S 1831 \mathrm{e}(\mathrm{d})(4)(\mathrm{A}),(\mathrm{C})$ (1994). The Federal Housing Finance Board allows a regulation to be modified if an NRSRO determines that changing a bond's provisions will not result in a ratings downgrade. See 12 C.F.R. § 910.6 (1993). The Housing and Urban Development Housing Finance Agency Requirements include a requirement that potential Housing Finance Agencies be rated "top tier" by an NRSRO and maintain an overall "A" rating for their bonds. See 24 C.F.R. § 266.100 (1994). The Student Loan Marketing Association has similar rules. See 20 U.S.C. § 1132f1 (1994). Ibid.

70 RICHARD CANTOR, FRANK PACKER, "The Credit Rating Industry", Op. Cit., p. 8.
} 
haciendo de esos procedimientos uno de los principales caballos de batalla para aquellos que demandan imperiosamente una mayor objetividad de las clasificaciones.

La capacidad de las agencias como proveedoras de unas valiosísimas "licencias calificatorias" no sólo situaron a las agencias en el centro del sistema financiero estadounidense, sino que redundó en una privatización de su Derecho, que entregaba a unos organismos privados una estratégica función pública, la determinación de los riesgos, de los que dependen no sólo las normas financieras de policía, sino las capacidades de apalancamiento financiero de las entidades.

\subsection{La exportación de su autoridad}

De este modo el principal problema para la soberanía monetaria de otros países vendría a través de la exportación de estas calificaciones allende de los mares, en el que las agencias servirían de una especie de "caballo de Troya", no sólo del modelo financiero, sino de los intereses estadounidenses. Este desembarco tendría lugar por dos vías, la primera y más sencilla vendría de manos de aquellos emisores no estadounidenses, con una relación directa con los mercados de deuda de aquel país (para la venta o cualquier otro negocio financiero), afectados automáticamente por cualquier cambio en las calificaciones ${ }^{71}$. Hay que tener en cuenta que la globalización financiera (es decir ese complejo fenómeno caracterizado por la disminución del control de los capitales por parte de los países, y la financiarización de la economía) generaría un gran mercado de bonos y títulos estadounidenses, al que no se resistirían los mercados primero occidentales y después de un gran número de países - que dotaría a esas calificaciones de repercusiones globales.

Pero la responsabilidad también sería compartida por otros países, que en su proceso de financiarización de la economía procederían a hacer uso de dichas calificaciones "importándolas" a su normativa. En Europa, no sólo las usarían los países anglosajones (la

\footnotetext{
71 FRANK PARTNOY, "The Siskel and Ebert of financial markets: two thumbs down for the credit rating agencies." Op. Cit., p. 730.
} 
«British Securities Industry Board» ${ }^{72}$ comenzaría a utilizar las calificaciones para la determinación del capital de las casas de valores británicas) sino que un país tan administrativista como Francia, las convertiría en instrumentos legales de la Comisión de Operaciones de la Bolsa para reducir los requisitos divulgativos. Su uso no sólo tuvo lugar en Europa, sino que por ejemplo en Japón, el Ministerio de Finanzas las utilizaría para autorizar a los emisores a vender deuda en sus mercados ${ }^{73}$. Por supuesto no dejarían de haber intentos de regulación internacional, como la que condujo a finales de 2009 a establecer un sistema de supervisión y registro de las Agencias de Calificación en todos los Estados del G-20 ${ }^{74}$, pero estos intentos serían muy tímidos y llegarían generalmente tarde.

Tal adquisición de poder (con su capacidad "normativa") centraría su actividad en la provisión de licencias, lo que se dejaría sentir en su capital reputacional, cometiendo importantes errores en sus predicciones, que demandarían pronto una nueva y más estricta regulación ${ }^{75}$.

\subsection{La estructuración de los bonos}

Pese al enorme reconocimiento que se había hecho del papel de las agencias, su marco regulatorio continuó siendo mínimo, lo que permitió una transformación de gran parte de sus

\footnotetext{
${ }^{72}$ La «British Securities Industry Board» sería en 2001 sustituida por la Autoridad de Servicios Financieros (FSA), que a su vez se dividiría en la «Financial Conduct Authority» y la «Prudential Regulation Authority», esta última como parte del Banco de Inglaterra.

${ }^{73}$ «375. See Cooper, supra note 161, at 51. Recently, however, regulators in countries outside the U.S. have begun regulating based on credit ratings. For example, the Japanese Ministry of Finance uses ratings to determine which issuers can sell debt in its markets; the French Commission des Opérations de Bourse uses ratings to reduce disclosure requirements; and the British Securities Industry Board uses ratings to determine the capital adequacy of British securities houses. See, e.g., McGuire, Ratings in Regulation, supra note 15, at 11 (non-U.S. generally); Rating the Rating Agencies, supra note 10, at 53 (same); The Use and Abuse of Reputation, supra note 10, at 18 (same); Harris, Inside Information for Outsiders, Financial Times, July 22, 1991, at 14 (same); Japan Set Up Council to Study Bond Ratings, REUTERS, Jan. 4, 1989 (Japan; copy on file with the author). Portugal has proposed legislation to allow only private companies with credit ratings to issue commercial paper. See Portugal Plans Own Version of CP Markets; Foreigners to be Excluded Initially, THOMSON'S INT'L BANKING REG., Mar. 16, 1992. For a general description of how countries other than the U.S. also incorporate credit ratings in their substantive financial market regulation, see Dale and Thomas, The Regulatory Use of Credit Ratings in International Financial Markets, J. INT'L SEC. MARKETS., Spring 1991, at 9». FRANK PARTNOY, "The Siskel and Ebert of financial markets: two thumbs down for the credit rating agencies." Op. Cit. p., 702.

${ }^{74}$ SIEGFRIED UTZIG, "The financial crisis and the regulation of credit rating agencies: A European banking perspective", Op. Cit. p. 10.

${ }^{75}$ Véase: Ibid., p. 703 y TIMOTHY M SULLIVAN, "Federal Preemption and the Rating Agencies: Eliminating State Law Liability to Promote Quality Ratings.", Minn. L. Rev., 94, 2009: p. 2136 - 2166, p. 2142.
} 
actividades. Así, a partir de servicios que seguirían siendo vistos como auxiliares, las agencias de calificación dejarían de ser meros "jueces" de la calidad de los bonos, para participar en su propia creación. A partir de los años setenta, las agencias de calificación - aunque evitarían denominar así la actividad - comenzarían a ayudar a estructurar las emisiones, en mucho mayor medida que lo habían hecho en el pasado $^{76}$, proporcionando ventajosos criterios de calificación y ayudando a los emisores a crear valores más negociables ${ }^{77}$, lo que en la práctica compromete su propia independencia (al "tener que auditar su propio trabajo" fuera realizado por ellas mismas realizando directamente la calificación, o bien recayera en alguna de su reducido círculo de agencias "colegas") ${ }^{78}$. Los problemas de conflicto de intereses quedarían así servidos, al existir incentivos distorsionadores de las calificaciones ${ }^{79}$. En realidad, el trabajo de las agencias se subdividió entre dos tipos de calificaciones muy distintas, por un lado, ofreciendo indicadores claros $\mathrm{y}$ aceptados internacionalmente sobre bonos corporativos $\mathrm{o}$ gubernamentales $^{80}$, y por otro estructurando productos extraordinariamente complejos, en la que la confianza en las agencias ocupaba una posición más que crucial ${ }^{81}$.

\section{LA CRISIS MUESTRA DOS ENFOQUES REGULATÓRIOS}

\footnotetext{
76 «The structured finance transactions that paved the way for subprime securities began in earnest in the 1970s. While the rating agencies' task was a challenging one, the pool of assets (receivables) at issue in the early transactions consisted of real estate mortgages made to prime borrowers, an asset whose performance over time was quite well understood. As time went on, different types of assets have been securitized; some asset classes have also become quite well understood. 3 Where that is the case, the transaction structuring becomes straightforward-the transactions are referred to as "cookie cutter"». CLAIRE HILL, Why Did Rating Agencies Do Such a Bad Job Rating Subprime Securities?, 71 U. Pitt. L. Rev. 585, 2010, p. 589. Disponible en Internet (consulta 7 de febrero de 2017): http://scholarship.law.umn.edu/faculty_articles/80.

77 TIMOTHY M SULLIVAN, "Federal Preemption and the Rating Agencies: Eliminating State Law Liability to Promote Quality Ratings.", Op. Cit., p. 2143.

${ }^{78}$ FREDERIC S MISHKIN, "Policy remedies for conflicts of interest in the financial system.", Paper presented to the Macroeconomics, monetary policy and financial stability conference. Ottawa: Bank of Canada, 2003. GINEVRA MARANDOLA, TIMOTHY SINCLAIR, "Credit Rating Agencies: A Constitutive and Diachronic Analysis", Sheffield Political Economy Research Institute Paper n ${ }^{\circ}$ 16, October 2014, p. 8. Disponible en Web (consulta 7 de febrero de 2017): http://speri.dept.shef.ac.uk/papers/

${ }^{79}$ GINEVRA MARANDOLA, TIMOTHY SINCLAIR, "Credit Rating Agencies: A Constitutive and Diachronic Analysis", Op. Cit. p. 8.

${ }^{80}$ SIEGFRIED UTZIG, "The financial crisis and the regulation of credit rating agencies: A European banking perspective", Op. Cit. p. 1.

81 «In the markets for structured products, by contrast, the role of the CRAs goes far beyond eliminating information asymmetry. Markets for structured products could not have developed without the quality assurance provided by CRAs to unsophisticated investors about inherently complex financial products. CRAs have operated as trusted gatekeepers. However, the ratings for structured credit turned out to be much less robust predictors of future developments than were the ratings for traditional single name securities». Ibid.
} 
Las experiencias de la crisis de deuda de 1997 afectaron a la credibilidad de las agencias, lo que llegó incluso a los parlamentos, donde las críticas a su actividad fueron muy encendidas $^{82}$, pero no fue hasta 2004 - tras los asuntos Enron ${ }^{83}$ y World.com - que la Organización Internacional de Comisiones de Valores (IOSCO-OICV) ${ }^{84}$ publicó un Código de Conductas Fundamentales, en las que se privilegiaba un enfoque autorregulatorio, que fue suscrito por las principales agencias ${ }^{85}$. Este Código establecía un conjunto de normas sobre la transparencia del proceso de calificación y supervisión, ciertas garantías para los procedimientos internos, y otras sobre la independencia y la ausencia de conflictos de intereses. El trabajo del IOSCO proporcionaba un marco normativo internacionalmente aceptado, pero no contenía ni aspectos metodológicos, ni un mecanismo sancionador ${ }^{86}$. Aunque las agencias de calificación suscribieron el Código voluntariamente decidieron también no abandonar la prestación de unos servicios auxiliares, que no eran neutrales con respecto a su actividad

\footnotetext{
82 «"Credit rating agencies are the biggest uncontrolled power in the global financial system, and thus in the national financial system too," said the President of the Federal Financial Supervisory Authority (BaFin) as early as 2003 at a hearing before the German parliament's finance committee (Deutscher Bundestag 2003)». Ibid. p. 4.

${ }^{83} \mathrm{El}$ asunto ENRON tuvo una gran trascendencia puesto que puso sobre el tapete como habían tenido lugar con absoluta impunidad un gran número de manipulaciones del mercado. Véase: McLEAN, PETER ELKIND, "Enron: The Smartest Guys in the Room: The amazing rise and scandalous fall of Enron", Portfolio Trade, 2005, y la película documental del mismo título: ALEX GIBNEY (Dir), "Enron: The Smartest Guys in the Room", USA, 2929 Entertainment-HDNet Films, 2005. TIMOTHY M SULLIVAN, "Federal Preemption and the Rating Agencies: Eliminating State Law Liability to Promote Quality Ratings", Minn. Law. Review, n 94, 2009, p. 21362166, p. 2144.

${ }^{84}$ «The International Organization of Securities Commissions (IOSCO) is the international body that brings together the world's securities regulators and is recognized as the global standard setter for the securities sector. IOSCO develops, implements and promotes adherence to internationally recognized standards for securities regulation. It works intensively with the G20 and the Financial Stability Board (FSB) on the global regulatory reform agenda. (...) IOSCO was established in 1983. Its membership regulates more than $95 \%$ of the world's securities markets in more than 115 jurisdictions; securities regulators in emerging markets account for $75 \%$ of its ordinary membership. The IOSCO Objectives and Principles of Securities Regulation have been endorsed by both the G20 and the FSB as the relevant standards in this area. They are the overarching core principles that guide IOSCO in the development and implementation of internationally recognized and consistent standards of regulation, oversight and enforcement. They form the basis for the evaluation of the securities sector for the Financial Sector Assessment Programs (FSAPs) of the International Monetary Fund (IMF) and the World Bank. By providing high quality technical assistance, education and training, and research to its members and other regulators, IOSCO seeks to build sound global capital markets and a robust global regulatory framework». IOSCOInternational Organization of Securities Commissions. Disponible en Web (consulta 26 de enero de 2017): https://www.iosco.org/

${ }^{85}$ SIEGFRIED UTZIG, "The financial crisis and the regulation of credit rating agencies: A European banking perspective", ADBI working paper series, Asian Development Bank Institute, Tokio, No. 188, 2010., p. 1 Disponible en Web (consulta 10 de diciembre de 2016): http://hdl.handle.net/10419/53684 http://www.adbi.org/working-paper/2010/01/26/3446.credit.rating.agencies.european.banking/

${ }^{86}$ SIEGFRIED UTZIG, "The financial crisis and the regulation of credit rating agencies: A European banking perspective", Op. Cit., p. 7.
} 
principal, ya que afectaba a su fiabilidad; una cuestión subestimada por las autoridades europeas y estadounidenses ${ }^{87}$. El Código llevó a la Unión Europea a aplazar sus intenciones regulatorias, tal y como se acordó por la Comisión Europea y el Comité de Reguladores Europeos de Valores - CESR ${ }^{88}$, por lo que se limitó a vigilar el grado del cumplimiento del acuerdo ${ }^{89}$. Al otro lado del atlántico, el Congreso estadounidense sí que decidiría regularlas, lo que haría a través de la Ley de Revisión de las Agencias de Calificación de Crédito de $2006^{90}$, lo que facilitaría su reconocimiento como Organizaciones de Calificación Estadística Nacionalmente Reconocida, sujetándola a la introducción de importantes requisitos divulgativos, como la publicación de sus metodologías de calificación, o cualquier conflicto de intereses ${ }^{91}$, pero sin que se exigiera ninguna metodología calificatoria ${ }^{92}$. Con la crisis de 2007 , los errores y conflictos de intereses quedaron al descubierto, lo que provocó una llamada generalizada a la regulación, como puso de manifiesto el informe del Foro de Estabilidad Financiera del 7 de abril, que les achacaba deficiencias metodológicas ${ }^{93}$, o el del Comité Issing del Gobierno alemán preparatorio para el G-20, que hacía lo propio con los conflictos de intereses ${ }^{94}$. La propia cumbre del G-20 (noviembre de 2008 en Washington) llamó a su mayor regulación ${ }^{95}$, anunciando una fuerte supervisión de acuerdo con un fortalecido código internacional de conducta ${ }^{96}$. El mismo año, el Tribunal Federal de Justicia (FCJ) de Australia condenó a Standard \& Poor's (S\&P), junto a otras dos entidades de inversión de capital, a indemnizar a trece ayuntamientos por recomendarles un producto financiero que les provocó pérdidas millonarias, al forzar su calificación crediticia ${ }^{97}$.

\footnotetext{
${ }^{87}$ GINEVRA MARANDOLA, TIMOTHY SINCLAIR, "Credit Rating Agencies: A Constitutive and Diachronic Analysis", Sheffield Political Economy Research Institute Paper, nº 16, October 2014, p. 8.

${ }^{88}$ SIEGFRIED UTZIG, "The financial crisis and the regulation of credit rating agencies: A European banking perspective", Op. Cit., p. 1.

${ }^{89}$ Ibid., p. 8.

${ }^{90}$ TIMOTHY M SULLIVAN, "Federal Preemption and the Rating Agencies: Eliminating State Law Liability to Promote Quality Ratings", Op. Cit., p. 2143.

${ }^{91}$ Ibid, p. 2144.

${ }^{92}$ Ibid., p. 2145.

${ }^{93}$ SIEGFRIED UTZIG, "The financial crisis and the regulation of credit rating agencies: A European banking perspective", Op. Cit., p. 2.

${ }^{94}$ Ibid., p. 3.

${ }^{95}$ Ibid., p. 0 y 1.

${ }^{96}$ Ibid., p. 9.

97 "Histórica sentencia contra Standard and Poor's en Australia", EUROPA PRESS, Sidney 05/11/2012 07:56. Disponible en Web (consulta 10 de febrero de 2017): http://www.publico.es/dinero/444893/historica-sentenciacontra-standard-and-poor-s-en-australia
} 
El principal escollo para un enfoque regulatorio común entre Europa y los Estados Unidos se centraba en las metodologías de la clasificación, puesto que representaban dos perspectivas muy distintas sobre el papel de las agencias. Si en Europa se reclamaba una regulación internacional, en Estados Unidos se seguía defendiendo su independencia y se prohibían las interferencias de la «SEC» en las metodologías (Art. 15 E (2) de la «Securities Exchange Acts of $1934 »)^{98}$. No obstante, la magnitud de los problemas llevaría en 2008 a la «SEC» a supervisar los requisitos de divulgación (las agencias serían responsables del cumplimiento de sus propios estándares) y a adquirir otros poderes de supervisión del uso de la información confidencial y los conflictos de intereses ${ }^{99}$. Finalmente, la «Dodd-Frank Wall Street Reform and Consumer Protection Act» autorizó una regulación de las metodologías de calificación crediticia, y creó un nuevo estándar para gobernar la responsabilidad privada de las agencias ${ }^{100}$ que no tenía precedentes. La ley establecía que "la Comisión [la «SEC»] establecerá normas para la protección de los inversores y el interés público en relación con los procedimientos y metodologías, incluidos los datos y modelos cualitativos y cuantitativos utilizados por las organizaciones de calificación estadística reconocidas a nivel nacional"101.

No obstante, y pese a estos avances reguladores, la jurisprudencia estadounidense volvería a legitimar a las agencias como meras empresas de información financiera, algo así como una especie de prensa especializada. El caso del Banco Comercial de Abu Dhabi contra Morgan Stanley \& Co. del Tribunal de Distrito de los Estados Unidos para el Distrito Sur de Nueva York de 2009 reafirmó que, bajo "circunstancias típicas", las agencias estarían bajo la robusta protección de la Primera Enmienda ${ }^{102}$, excepto cuando "diseminan sus calificaciones a

\footnotetext{
98 Ibid. p. 8.

99 Ibid., p. 7 y 8.

100 CALEB DEATS, "Talk that isn't cheap: Does the first amendment protect credit rating agencies' faulty methodologies from regulation?", Columbia Law Review, 2010, p. 1818-1864, p. 1862.

101 «"(r) CREDIT RATINGS METHODOLOGIES. - The Commission shall prescribe rules, for the protection of investors and in the public interest, with respect to the procedures and methodologies, including qualitative and quantitative data and models, used by nationally recognized statistical rating organizations that require each nationally recognized statistical rating organization-». Dodd-Frank Wall Street Reform and Consumer Protection Act (Pub.L. 111-203, H.R. 4173. Disponible en Internet (consulta 9 de febrero de 2017): https://www.congress.gov/bill/111th-congress/house-bill/4173

102 «Amendment I. Congress shall make no law respecting an establishment of religion, or prohibiting the free exercise thereof; or abridging the freedom of speech, or of the press; or the right of the people peaceably to assemble, and to petition the government for a redress of grievances.». US Constitution, Legal Information Institute - Cornell University Law School, Disponible en Internet (consulta 9 de febrero de 2017): https://www.law.cornell.edu/constitution/first_amendment.
} 
un selecto grupo de inversionistas"103. De este modo, salvo "cuando una agencia calificadora ha difundido sus calificaciones a un selecto grupo de inversionistas, en vez de al público en general, en que la agencia de calificación no cuenta con la protección típica de la Primera Enmienda", y precisamente por no constituir una cuestión de interés público ${ }^{104}$, las calificaciones tendrían el mismo tipo de protección a la libre expresión que se otorga a la opinión periodística, con la consideración de una calificación de bonos como un editorial o "una opinión basada en una educada conjetura sobre el futuro" ${ }^{105}$. De este modo, las predicciones de las agencias serían como las editoriales de los periódicos o las clasificaciones que estos hacen de las universidades, y como tales estarían protegidas por la Primera Enmienda, sobre la base de que "una declaración errónea es inevitable en el debate libre, y ... debe ser protegida para que las libertades de expresión tengan el "espacio de respiración" que necesitan..." (N.Y. Times Co. v. Sullivan, 376 US 254, 271 - 72 (1964) (citando NAACP v. Button, 371 US 415, 433 $(1963))^{106}$.

Esta jurisprudencia suponía un reto ante los avances que la ley de consumidores había logrado en torno a la problemática cuestión de la metodología, que se basaba en la consideración de las clasificaciones como una cuestión de interés público, y no de meras opiniones privadas ${ }^{107}$. Sin embargo, la brecha abierta por esta sentencia ponía en riesgo una parte de dicha legislación, y podía generar una nueva normativa sobre la responsabilidad de las agencias frente a los inversionistas ${ }^{108}$. No obstante, su protección sería menor que la de otras manifestaciones de la libertad de expresión, ya que, a la luz del resto de la jurisprudencia, las calificaciones deberían

${ }^{103}$ CALEB DEATS, "Talk that isn't cheap: Does the first amendment protect credit rating agencies' faulty methodologies from regulation?", Op. Cit. p. 1862.

${ }^{104}$ Ibid. p. 1822.

105 «Making an argument about the First Amendment, to begin with. Mr. Abrams will contend that S.\& P.'s ratings deserve exactly the sort of free-speech protections afforded to journalists, on the theory that a bond rating is like an editorial - an opinion based on an educated guess about the future. And for the same reason you can't sue editorial writers, Mr. Abrams will argue that you can't sue a bond rater because the economy went into a free fall that few saw coming». DAVID SEGAL, "A matter of opinion?", New York Times, 19 de Julio de 2009. Disponible en Internet (consulta 9 de febrero de 2017): http://www.nytimes.com/2009/07/19/business/19floyd.html

106 «Historically, the First Amendment has protected such communications, even in the form of advertisements, against charges of negligence on the grounds that "erroneous statement is inevitable in free debate, and ... must be protected if the freedoms of expression are to have the 'breathing space' that they 'need ... to survive. 5". (...) Note 5: N.Y. Times Co. v. Sullivan, 376 U.S. 254, 271-72 (1964) (quoting NAACP v. Button, 371 U.S. 415, 433 (1963)); see also Compuware Corp. v. Moody's Investors Servs., Inc., 499 F.3d 520, 525, 531 (6th Cir. 2007) (applying N.Y. Times's "actual malice" standard to defamation and breach of contract claims against rating agency)». CALEB DEATS, "Talk that isn't cheap: Does the first amendment protect credit rating agencies' faulty methodologies from regulation?", Op. Cit., p. 1819.

${ }^{107}$ Ibid., p. 1822.

${ }^{108}$ Ibid., p. 1819. 
ser consideradas como un mero discurso comercial ("no hacen más que proponer una transacción comercial") que "hace menos necesario tolerar declaraciones inexactas por miedo a silenciar al hablante" (véase la sentencia «State Bd. De Pharmacy v. Va. Citizens Consumer Council, Inc., 425 US 748, 762 (1976)») ${ }^{109}$. Una tensión entre la protección a la libertad de expresión y la regulación metodológica ${ }^{110}$ no resuelta ante la insuficiente jurisprudencia en los Estados Unidos ${ }^{111}$.

\section{LA NORMATIVA EUROPEA}

Este diferente enfoque, así como la dependencia foránea que venía generando para los europeos han generado una serie de propuestas como las del Comité Issing, que apostaban porque las calificaciones fueran monitoreadas por los reguladores; o las del Grupo Larosière, que en 2009 se dispusieron a proponer a la Comisión Europea una base para un futuro Reglamento de la $U^{112}$. Estos trabajos no eran meros brindis al sol, sino que respondían a la explícita declaración de los dirigentes de Francia, Alemania, Italia, Reino Unido y el Presidente de la Comisión Europea, que en un comunicado conjunto criticaron el papel de las agencias en los productos estructurados, y sus conflictos de intereses, proponiendo una regulación europea $^{113}$. En el Reino Unido, la Autoridad de los Servicios Financieros («FSA») apoyó los puntos principales del proyecto de Reglamento de la Unión Europea ${ }^{114}$, mientras que un optimista Parlamento Europeo debatía sobre como obligar a los inversores a utilizar las calificaciones emitidas por una hipotética agencia de calificación europea ${ }^{115}$. En noviembre de 2008 se publicó una propuesta de Reglamento de la UE, que el 23 de abril de 2009 fue aprobado por el Parlamento, y el 5 de mayo por el Consejo de Ministros ${ }^{116}$. La norma europea intentó abordar algunos de los aspectos más controvertidos de la actividad de las agencias, como la

\footnotetext{
${ }^{109}$ Ibid., p. 1820.

110 Ibid.

${ }^{111}$ Ibid., p. 1821.

112 SIEGFRIED UTZIG, "The financial crisis and the regulation of credit rating agencies: A European banking perspective", Op. Cit., p. 10.

113 Ibid., p. 9.

${ }^{114}$ Ibid., p. 11.

115 Ibid., p. 12.

116 Ibid., p. 15.
} 
cuestión de la licencia regulatoria, que establecía que las entidades de crédito sólo podían utilizar "con fines reglamentarios" aquellas calificaciones que hubieran sido emitidas por una Agencia registrada en la Unión Europea o que cumpliera con los criterios de equivalencia del Reglamento $^{117}$. Para el registro, la agencia establecía un mecanismo y un "colegio de supervisores", que permite a las registradas avalar las calificaciones realizadas por sus filiales fuera de la UE, siempre que estas operen bajo un régimen de supervisión no menos estricto, y exista una razón objetiva para que la calificación se realice en un Estado no miembro ${ }^{118}$. La preocupante cuestión de los productos estructurales también fue abordada, estableciendo que estos contarían con algún tipo de "símbolo adicional", y una mayor divulgación de información financiera como las pérdidas, el flujo de efectivo, y las simulaciones de estrés financiero realizadas $^{119}$. Incluso se estableció una restricción adicional a la emisión de calificaciones para productos complejos: "cuando la falta de datos fiables o la complejidad de la estructura de un nuevo tipo de instrumento financiero o la calidad de la información disponible no sea satisfactoria o plantea serias dudas sobre si una agencia de calificación crediticia puede proporcionar una calificación creíble, la agencia de calificación crediticia se abstendrá de emitir una calificación crediticia o retirar una calificación existente" ${ }^{120}$.

En noviembre de 2011 la Comisión europea presentó una iniciativa que se convertiría en una nueva normativa europea que entraría en vigor en $2013^{121}$ y que pretendía prevenir los riesgos del funcionamiento de las agencias que la crisis había descubierto. La nueva normativa no establecerá la exigencia de una metodología, pero al menos sí ciertas reglas de transparencia. Para ello, la Unión Europea creará una Plataforma Europea de Calificación Crediticia dependiente de la Autoridad Europea de Valores y Mercados (ESMA) en la que las agencias deberán publicar información relativa a sus evaluaciones, con objeto de que los interesados puedan llevar a cabo sus propios análisis y comparaciones ${ }^{122}$. Además, especialmente preocupados por los ratings soberanos no deseados, la normativa establecerá un calendario con

\footnotetext{
117 Ibid.

118 Ibid.

${ }^{119}$ Ibid., p. 16.

${ }^{120}$ Ibid.

${ }^{121}$ El Reglamento (UE) No 462/2013 del Parlamento Europeo y del Consejo el 21 de mayo 2013 que modifica el Reglamento (CE) No 1060/2009 sobre agencias de calificación crediticia y la Directiva 2013/14/UE del Parlamento Europeo y del Consejo el 21 de mayo 2013 que modifica la Directiva 2003/41/CE, Directiva 2009/65/CE y la Directiva 2011/61/UE.

122 GÓMEZ ACEBO \& POMBO, "Nueva legislación europea aplicable a las agencias de calificación crediticia", Análisis GA\&P, Bruselas, junio 2013, p. 1. Disponible en Web (consulta diciembre 2018): https://www.gap.com/en/publicaciones/nueva-legislacion-europea-aplicable-a-las-agencias-de-calificacion-crediticia/
} 
tres fechas para su publicación, siempre en viernes y tras el cierre de los mercados, y que deberán estar acompañados del respectivo informe explicativo ${ }^{123}$. Pero sin embargo, las principales novedades de la regulación será la posibilidad de que inversores y entidades calificadas puedan exigir la responsabilidad civil a las agencias, cuando de forma deliberada o negligente cometan importantes fallos en sus calificaciones; así como la incentivación de la contratación simultánea para así promover la contratación - como segunda - de agencias de menor tamaño, con una cuota inferior al $10 \%$ del mercado, que erosionen el oligopolio de las grandes agencias $^{124}$.

No obstante, estos cambios, Europa sigue sin contar con agencias de calificación crediticia importantes, de modo que la doctrina es consciente de que las expectativas sobre el impacto de la nueva normativa no deberían ser excesivamente elevadas ${ }^{125}$. Para ello, no debe de olvidarse que las calificaciones cuentan con aspectos denominados "cuantitativos", y otros que bien podemos denominar como "cualitativos" que significan en sí mismo una fuente del poder financiero internacional ${ }^{126}$, y en los que hay elementos tan subjetivos (o poco neutrales) como el denominado "riesgo político"127. En realidad, la mayor parte de los países ven sometidas sus políticas económicas a la autoridad de un pequeño puñado de firmas, cuya responsabilidad no es fácil de determinar y que determinan la ortodoxia económica a desarrollar, determinando los flujos internacionales de capital ${ }^{128}$.

\section{REFERÊNCIAS BIBLIOGRÁFICAS}

CALEB DEATS, "Talk that isn't cheap: Does the first amendment protect credit rating agencies' faulty methodologies from regulation?", Columbia Law Review, 2010, p. 1818-1864, p. 1862.

CHRISTOPHER M BRUNER y ABDELAL RAWI, "To judge Leviathan: sovereign credit ratings, national law, and the world economy", Journal of Public Policy 25.02, 2005; p. 191-

\footnotetext{
${ }^{123}$ Ibid.

${ }^{124}$ Ibid., p. 2.

${ }^{125}$ SIEGFRIED UTZIG, "The financial crisis and the regulation of credit rating agencies: A European banking perspective", Op. Cit., p. 18.

${ }^{126}$ CHRISTOPHER M BRUNER, ABDELAL RAWI, "To judge Leviathan: sovereign credit ratings, national law, and the world economy.", Op. Cit., p. 194.

${ }^{127}$ Ibid., p. 212.

${ }^{128}$ Ibid., p. 211.
} 
217, p. 192. Disponible en Web (consulta 13 de diciembre de 2016): https://www.cambridge.org/core/journals/journal-of-public-policy/article/div-classtitletojudge-leviathan-sovereign-credit-ratings-national-law-and-the-worldeconomydiv/25F0DE0339EF16349B5E2E517D5DA6A9

CLAIRE HILL, Why Did Rating Agencies Do Such a Bad Job Rating Subprime Securities?, 71 U. Pitt. L. Rev. 585, 2010, p. 589. Disponible en Internet (consulta 7 de febrero de 2017): http://scholarship.law.umn.edu/faculty_articles/80.

DE MIGUEL-DOMÍNGUEZ, JOSÉ CARLOS, al., "La medición del riesgo de crédito y el nuevo acuerdo de capital del Comité de Basilea", Publicación en Universidad de Santiago de Compostela, 2003, p. 1-21.

FRANK PARTNOY, "The Siskel and Ebert of financial markets: two thumbs down for the credit rating agencies." Washington University Law Quarterly Vol. 77, 1999, p: 619-712, p. 682. Disponible en Web (consulta 10 de diciembre de 2016): https://papers.ssrn.com/sol3/papers.cfm?abstract_id=167412

FREDERIC S MISHKIN, "Policy remedies for conflicts of interest in the financial system.", Paper presented to the Macroeconomics, monetary policy and financial stability conference. Ottawa: Bank of Canada, 2003.

GILBERT HAROLD, Bond ratings as an investment guide: An apraisal of their effectiveness, Columbia University, 1987. En: FRANK PARTNOY, "The Siskel and Ebert of financial markets: two thumbs down for the credit rating agencies." Washington University Law Quarterly Vol. 77, 1999, p: 619-712, p. 682. Disponible en Web (consulta 10 de diciembre de 2016): https://papers.ssrn.com/sol3/papers.cfm?abstract_id=167412

GINEVRA MARANDOLA, TIMOTHY SINCLAIR, "Credit Rating Agencies: A Constitutive and Diachronic Analysis", Sheffield Political Economy Research Institute Paper $\mathrm{n}^{\mathrm{o}} 16$, October 2014, p. 8. Disponible en Web (consulta 7 de febrero de 2017): http://speri.dept.shef.ac.uk/papers/

GÓMEZ ACEBO \& POMBO, "Nueva legislación europea aplicable a las agencias de calificación crediticia", Análisis GA\&P, Bruselas, junio 2013. Disponible en Web (consulta diciembre 2018): https://www.ga-p.com/en/publicaciones/nueva-legislacion-europeaaplicable-a-las-agencias-de-calificacion-crediticia/

HERWIG M. LANGOHR, PATRICIA T. LANGOHR, The Rating Agencies and their Credit Ratings What They Are, How They Work and Why They Are Relevant, Vol. 510. John Wiley \& Sons, 201, p. 376.

JOHN MAYNARD KEYNES, The General Theory of Employment, Interest and Money, En su "Chapter 13, The General Theory of the rate of interest», University of Missouri-Kansas City. Disponible en Web (consulta 18 de enero de 2017): http://www.umkc.edu/umkcsearch/?cx $=008281657408603500330 \% 3$ Avpif $2 \mathrm{cmpa} 14 \&$ cof $=$ FORID $\% 3 A 10 \&$ ie $=$ UTF-

$\underline{8 \& q=k e y n e s}$ 
PATRICK VAN ROY, "Credit ratings and the standardised approach to credit risk in Basel II", European Central Bank, Working Paper Series $n^{\circ}$ 517, August 2005, p. 5. Disponible en Web (consulta 10 de febrero de 2017): http://www.ecb.europa.eu/pub/pdf/scpwps/ecbwp517.pdf

PAUL KRUGMAN, "S\&P and the USA", The Conscience of a Liberal, New York Times, 5 de agosto de 2011. Disponible en Web (consulta 14 de diciembre de 2016): http://krugman.blogs.nytimes.com/2011/08/05/sp-and-the-usa/?smid=tw-

NytimesKrugman\&seid=auto

RAÚL ROJAS SORIANO, Investigación social: Teoría y práxis, Plaza y Valdés, México, 2007. p. $97-98$.

RAWI ABDELAL, Capital Rules, The construction of Global Finance, Harvard University Press, Cambridge (Massachusetts-US) and London (UK), 2007.

RAWI ABDELAL y BRUNER, "Private Capital and Public Policy: Standard \& Poor's Sovereign", Credit Ratings. Harvard Business School Case No. 9-705-027, 2005.

RICHARD CANTOR, FRANK PACKER, "The Credit Rating Industry", FRBNY Quarterly Review, Summer-Fall 1994, p. 1-26.

SIEGFRIED UTZIG, "The financial crisis and the regulation of credit rating agencies: A European banking perspective", ADBI working paper series, Asian Development Bank Institute, Tokio, No. 188, 2010, p. 9. Disponible en Web (consulta 10 de diciembre de 2016): http://hdl.handle.net/10419/53684

SYLLA RICHARD, "A Historical Primer on the Business of Credit Ratings." Op. Cit., p. 5.

TIMOTHY M SULLIVAN, "Federal Preemption and the Rating Agencies: Eliminating State Law Liability to Promote Quality Ratings.", Minn. L. Rev., 94, 2009: p. 2136 - 2166, p. 2142.

Submetido em: 05/07/2019

Aceito em: 24/07/2019 